\title{
sciendo
}

\section{A Comparison Between The Recovery Responses Following an Eccentrically Loaded Bench Press Protocol Vs. Regular Loading in Highly Trained Men}

\author{
by \\ Sandro Bartolomei ${ }^{1}$, Valentina Totti ${ }^{1}$, Federico Nigro ${ }^{1}$, Simone Ciacci ${ }^{1}$, \\ Gabriele Semprini ${ }^{1}$, Rocco Di Michele ${ }^{1}$, Matteo Cortesi², Jay R. Hoffman ${ }^{3}$
}

The purpose of this study was to compare the physiological responses of a single bout of an eccentric accentuated bench press protocol (120\% of 1RM in the eccentric phase/80\% in the concentric phase; [120/80]) versus a regular high-intensity exercise protocol $(80 \% / 80 \%$; [80/80]) in resistance-trained men. Eleven men (age $=25.6 \pm 3.9 \mathrm{y}$; body mass $=84.6 \pm 11.2 \mathrm{~kg}$; body height $=176.4 \pm 3.9 \mathrm{~cm}$ ) with $6.3 \pm 3.4$ y of resistance training experience performed each protocol in counterbalanced, randomized order. Isometric, isokinetic and ballistic tests were performed at the bench press (IBPF, ISOK and BTP, respectively) at baseline (BL), 15-min (15P), 24-h (24P), and 48-h (48P) post-exercise for each testing session. In addition, muscle thickness of the pectoralis major (PecMT) was measured at the same timepoints via ultrasound. Significantly greater reductions in BTP $(p<0.001)$, peak force during both ISOK $(p=$ $0.005)$ and IBPF $(p=0.006)$ at $15 P$ were detected in $120 / 80$ compared to $80 / 80$. BTP was still significantly $(p=0.009)$ impaired at $48 \mathrm{P}$ following the 120/80 protocol, while no differences were noted following 80/80. PecMt was significantly elevated following both 120/80 and 80/80 ( $p<0.05)$ at 15P, but significant differences between the trials were present at $15 P$ and $24 P(p=0.005$ and $p=0.008$, respectively). Results indicated that heavy eccentric loading during the bench press exercise caused greater performance deficits than a bout of traditionally loaded high intensity resistance exercise. Power performance appears to be more influenced by the 120/80 protocol than isometric peak force. Eccentrically loaded exercise sessions should be separated by at least 48 hours to obtain a complete recovery of the initial muscle morphology and performance.

Key words: eccentric, muscle architecture, strength.

\section{Introduction}

It is well known that heavy resistance training can result in significant skeletal muscle hypertrophy and in maximal strength and power improvements (Bartolomei et al., 2018). The use of eccentric training, characterized by a greater external load applied during the eccentric phase of the lift, and removed before the concentric phase, has been shown to provide an advantage in strength adaptations compared to traditional loading paradigms (Walker et al., 2016). Athletes competing in strength and power events and interested in maximizing muscle mass indeed, often include heavy negative sets (also known as high intensity eccentric exercise) in their training routines to promote further muscle adaptations (Schoenfeld and Grgic, 2017).

Eccentric exercise has been associated with muscle damage resulting in significant increases

1 - Department of Biomedical and Neuromotor Sciences, University of Bologna, 40100 Bologna, Italy.

2 - Department for Life Quality Studies, University of Bologna, C.so D'Augusto 237, 47921, Rimini, Italy.

3 - College of Education and Human Performance, University of Central Florida, Orlando, FL 32766, USA. 
in circulating concentrations of creatine kinase and myoglobin (Lieber et al., 2002), reduction in muscle performance (Sayers and Clarkson, 2001) and change in muscle morphology (Gordon et al., 2017). Muscle function has been recognized as one of the most valid indicators for quantifying the magnitude of exercise-induced muscle damage following both eccentric (Sayers and Clarkson, 2001) and concentric/eccentric exercise (Bartolomei et al., 2017). Acute effects of exercise induced muscle damage appear particularly evident in dynamic performances, such as power produced at light loads, than maximal isometric strength (Sakamoto et al., 2010) or isokinetic peak torque expressed at slow speeds of contraction (Abaïdia et al., 2017). Recently, changes in muscle morphology following high-volume eccentric exercise focused on the lower body have been detected by B-mode ultrasound images (Gordon et al., 2017). Some of these studies though, were carried out using isokinetic dynamometers in untrained individuals (Schoenfeld and Grgic, 2017). Furthermore, significant increases in blood concentrations of IL-6 and C-reactive protein (biomarkers of the inflammatory response), have been detected following high-volume resistance exercise sessions (Bartolomei et al., 2017). It has been well established that attenuation of muscle damage and functional decline following eccentric exercise may occur in trained individuals compared to detrained or untrained individuals (McHugh, 2003).

To the best of our knowledge, no scientific studies to date have investigated the recovery phase following an accentuated eccentric exercise session performed in the bench press in highlytrained individuals. Thus, the aim of the present study was to compare the acute effects and the recovery response following an accentuated eccentric bench press protocol and a traditional high intensity protocol on performance and muscle architecture of the pectoral muscle in highly trained males. It was hypothesized that an eccentrically loaded bench press session would result in greater changes in maximal strength, power and muscle morphology than a regular concentric/eccentric bench press session. More insight about the recovery process following eccentrically loaded bench press may help coaches and practitioners to better periodize eccentric loads for optimal stimulation of muscle strength.

\section{Methods}

Participants

Eleven experienced, resistance trained men $($ Mean \pm SD: age $=25.6 \pm 3.9 \mathrm{y} ;$ body mass $=84.6 \pm$ $11.2 \mathrm{~kg}$; body height $=176.4 \pm 3.9 \mathrm{~cm}$; body fat composition $=12.3 \pm 5.8 \%$; resistance training experience $=6.3 \pm 4.5 \mathrm{y} ; 1-\mathrm{RM}$ bench press $=115.8$ $\pm 16.7 \mathrm{~kg}$ ) volunteered to participate in the present study. Participants were not permitted to use any additional dietary supplementation and were asked if they had ever used performance enhancing drugs. Participants that responded "yes" were not allowed to take part in the present study. The study was approved by the Ethical Committee of the University of Bologna.

Measures

Prior to 1-RM bench press testing, participants performed a standardized warm-up described in previous studies (Bartolomei and Hoffman, 2018). The 1-RM test for the bench press was performed on a Smith machine using methods previously defined by Hoffman (2014).

During each visit, participants were required to perform a bench press throw power test (BTP), an isokinetic bench press test at $75 \mathrm{~cm} / \mathrm{s}$ and at 25 $\mathrm{cm} / \mathrm{s}$ (ISOK75 and ISOK25, respectively), and an isometric bench press force test (IBPF). Each participant performed two attempts for each test with a 3 min recovery time and the highest values measured were recorded for further analysis.

The BTP test was performed using a Smith machine. Participants laid down on a bench in the supine position with the bar on their chest. They were instructed to push as explosively as possible and to throw the bar as high as possible. Two spotters decelerated the bar during the descending phase. Participants pressed loads corresponding to $30 \%$ and $50 \%$ of their 1-RM (BTP30 and BTP50, respectively). An optical encoder (Tendo Unit model V104, Tendo Sports Machines, Trencin, Slovak Republic) measured the mean power (BTP) expressed by the participants. Intraclass coefficients were determined to be $0.94(\mathrm{SEM}=16.3 \mathrm{w})$ and 0.95 $(\mathrm{SEM}=13.6 \mathrm{w})$ for BTP30 and BTP50, respectively.

Isokinetic bench press strength measurements were performed following the BT using a linear isokinetic dynamometer (Lido Loredan Linea, Shirley, NY). Participants were positioned with their elbows at $90^{\circ}$ of flexion on a bench and their grip width was measured and 
recorded. Isokinetic concentric measurements were performed at a velocity of $75 \mathrm{~cm} / \mathrm{s}$ (ISOK75) and $25 \mathrm{~cm} / \mathrm{s}$ (ISOK25). Intraclass coefficients were $0.90(\mathrm{SEM}=37.8 \mathrm{~N})$ and $0.92(\mathrm{SEM}=39.5 \mathrm{~N})$ for ISOK75 and ISOK25, respectively.

A $6 \mathrm{~s}$ isometric bench press force (IBPF) assessment was also performed using a power rack that permitted fixation of the bar. The bench was positioned over a force plate (Kistler 9260, $500 \mathrm{~Hz}$, Winterthur, Switzerland Kinsler, 500Hz). Participants were required to position themselves on the bench with their elbows at $90^{\circ}$ of flexion and with hips and knees at $90^{\circ}$ of flexion. Participants were not permitted to position the feet on the ground. Grip width was measured to reproduce the same position for all testing sessions. The force expressed against the bar was transmitted by the bench to the force plate and the peak force (IBPF) was measured. The intraclass coefficient was $0.89(\mathrm{SEM}=85.5 \mathrm{w})$ for IBPF.

Non-invasive skeletal muscle ultrasound images were collected from the participant's left side. Pectoralis muscle thickness (PecMT) was measured at the site between the third and the fourth intercostal under the clavicle midpoint (Abe et al., 2000). Measurement required participants to lay in a supine position for a minimum of 15 min before images were collected. The same investigator performed all landmark measurements for each participant. A $12 \mathrm{MHz}$ linear probe scanning head (Echo Wave 2, Telemed Ultrasound Medical System, Milan, Italy; gain $=50 \mathrm{~dB}$; image depth $=5 \mathrm{~cm}$ ) was used to collect all ultrasound images. Three consecutive PecMT images were captured and analyzed. For each image, MT was measured with a single perpendicular line from the superficial aponeurosis to the deep aponeurosis. Three different images of the same muscle were taken at the same place and measured. The average of these three MT measures was used for statistical analyses. The intra-class correlation coefficient (ICC) for the MT measures was 0.95 (SEM = 1.05 $\mathrm{mm})$.

\section{Design and Procedures}

Figure 1 depicts the experimental design of this investigation (counterbalanced crossover design). Participants were requested to report to the laboratory on seven separate occasions. During the first visit, participants were assessed for one-repetition maximal strength (1-RM) on the bench press exercise and for anthropometric measures. Body measurements included body mass, height, and body composition. The body fat percentage was estimated from skinfold caliper measures using the method of Evans et al. (2005). The same investigators, with intraclass coefficients between 0.98 and 0.99 , performed all skinfold analysis assessments. Participants reported back to the laboratory at least 72-h following their initial visit and performed the exercise training session. A 5-day washout period was introduced between the first and the second trial of the counterbalanced crossover design. During this period, participants were not allowed to perform any upper-body exercises. Immediately prior to the exercise session (Baseline; BL) strength and power assessments (BTP30, BTP50, ISOK75, ISOK25 and IBPF) were performed. Following the workout, participants were tested 15-min (15P), 24-h (24P) and 48-h (48P) post-exercise. Pectoralis major muscle ultrasonography was obtained at each time point.

Participants were asked to perform six sets of five repetitions at $80 \%$ of the previously measured 1-RM using the same load in both the concentric and the eccentric phase $(80 / 80)$ or six sets of five repetitions at $80 \%$ of $1-\mathrm{RM}$ in the concentric phase and $120 \%$ of $1-\mathrm{RM}$ in the eccentric phase $(120 / 80)$. The aforementioned exercise protocols were based on the recommendations for resistance training of weightlifters and strength and power athletes (Hori et al., 2005). In 120/80, the eccentric load was applied by two spotters at the end of the concentric phase and removed at the end of the eccentric phase when the bar was supported by the damping springs of the smith machine (Figure 2). Participants were instructed to spend $3 \mathrm{~s}$ during the eccentric phase in both $80 / 80$ and $120 / 80$ trials. If the participants were not able to keep the eccentric contraction for $3 \mathrm{~s}$ or lift the bar in the concentric phase, then the load was reduced by $5 \%$ of the load on the bar. Participants were asked to push as explosively as possible during the concentric phase. Recovery time between sets was $2 \min 30 \mathrm{~s}$.

Statistical analysis

A Shapiro-Wilk test was used to assess the normal distribution of the data. Performance and muscle architecture data were analyzed using a two factor (trial $\times$ time) analysis of variance (ANOVA) with repeated measures. In the event of 
a significant $\mathrm{F}$ ratio, dependent $t$-tests with a Bonferroni adjustment were used to examine pairwise comparison between trials for each time point. In the event of a significant trialxtime interactions each group was analyzed separately by a one factor ANOVA with repeated measures on time. Significance was accepted at an alpha level of $p \leq 0.05$, and all data are reported as mean \pm SD.

\section{Results}

Results for strength and power isokinetic measures are depicted in Table 1. A significant trial $\times$ time interaction was found for BTP30 $(\mathrm{F}=$ 14.949; $\left.p=0.001 ; \eta^{2}=0.651\right)$. A significant trial difference in BTP30 (Figure 3) was noted at 15P ( $p$ $<0.001)$ and 48P $(p=0.010)$. During $120 / 80$, BTP30 performance was significantly reduced from baseline (BL) at 15P $(-12.9 \% ; p<0.001)$ and $48 \mathrm{P}(-$ $4.3 \% ; p=0.006)$, while during $80 / 80$, BTP30 was not significantly $(p>0.05)$ reduced at any of the time points.

Significant interactions were also noted for ISOK75 ( $\left.\mathrm{F}=6.710 ; p=0.025 ; \eta^{2}=0.528\right)$, ISOK25 $\left(\mathrm{F}=6.569 ; p=0.015 ; \eta^{2}=0.523\right)$ and $\operatorname{IBPF}(\mathrm{F}=3.708$; $p=0.034 ; \eta^{2}=0.346$ ). Pairwise comparisons indicated significant differences in ISOK75 between $120 / 80$ and $80 / 80$ at 15P ( $p=0.005)$. ISOK75 was significantly reduced from BL at $15 \mathrm{P}$ $(-21.4 \% ; p=0.001), 24 \mathrm{P}(-11.0 \% ; p=0.043)$ and $48 \mathrm{P}$ $(-6.3 \% ; p=0.009)$ following the $120 / 80$ protocol. ISOK75 was significantly reduced from BL in $80 / 80$ at $15 \mathrm{P}$ only $(-8.2 \% ; p=0.028)$. Significant differences between $120 / 80$ and $80 / 80$ were detected at 15P for ISOK25 and IBPF ( $p=0.002$ and $p=0.006$, respectively). Decrements in performance from BL during 120/80 were significant at 15P and 24P for ISOK25 $(-19.8 \% ; p=$ 0.001 and $-9.4 \% ; p=0.005$, respectively). IBPF was significantly reduced from BL in $120 / 80$ at $15 \mathrm{P}$ only $(-8.8 \% ; p<0.001)$. ISOK25 and IBPF performances in $80 / 80$ were not significantly different from BL at any time point $(p>0.05)$. No significant interactions were observed for BTP50 $\left(\mathrm{F}=2.799 ; p=0.092 ; \eta^{2}=0.286\right)$. However, a significant main effect for time was noted $(\mathrm{F}=$ 13.404; $\left.p=0.001 ; \eta^{2}=0.657\right)$. Furthermore, main effects for time were seen for BTP30 ( $F=18.750$; $\left.p<0.001 ; \eta^{2}=0.701\right)$, ISOK25 $(\mathrm{F}=16.131, p<0.001$; $\left.\eta^{2}=0.729\right)$, ISOK75 ( $\left.\mathrm{F}=14.314, p=0.001 ; \eta^{2}=0.705\right)$ and IBPF $\left(F=14.088, p<0.001 ; \eta^{2}=0.668\right)$.
Changes in ultrasound measures can be observed in Figure 4. Significant trial $\times$ time interactions were found for PecMT ( $\mathrm{F}=3.999 ; p=$ 0.031; $\left.\eta^{2}=0.286\right)$. Significant increases from BL were observed for PecMT at $15 \mathrm{P}$ in both $120 / 80$ $(+15.1 \% ; p=0.001)$ and $80 / 80(+6.9 \% ; p=0.038)$. The increase in PecMT, however, was significantly greater following 120/80 compared to 80/80. Pairwise comparisons also revealed significant differences between the groups for PecMT at 15P $(p=0.005)$ and at 24P $(p=0.008)$. Examples of Pec ultrasonography images collected on a participant at $\mathrm{BL}$ and $15 \mathrm{P}$ following both $120 / 80$ and $80 / 80$ are provided in Figure 5a, $5 b$ and 5c, respectively.

\section{Discussion}

In this study, an accentuated eccentric upperbody strength training session elicited greater strength and power decrements compared to a traditional high intensity exercise session, controlled for repetitions performed, in experienced strength-trained men. Acute recovery appeared to be more negatively affected by the accentuated eccentric loads, especially in performances requiring high movement speeds compared to isometric assessments. In particular, isokinetic force expressed at $75 \mathrm{~cm} / \mathrm{s}$ and power expressed at $30 \%$ of $1-\mathrm{RM}$ appeared more influenced by the $120 / 80$ exercise protocol than isokinetic force at $25 \mathrm{~cm} / \mathrm{s}$, power at $50 \%$ of $1-\mathrm{RM}$ and isometric peak force. This is consistent with previous studies that found greater negative effects of muscle damage on dynamic strength expressions compared to isometric force (Sakamoto et al., 2010; Skurvydas et al., 2011). Some authors have suggested that high intensity eccentric contraction may be associated with a preferential activation of fast twitch motor units (Vogt and Hoppeler, 2014). The selective recruitment of fast twitch motor units during the eccentric phase may concentrate the mechanical stress on these type II muscle fibers, leading to sarcomere disruption and membrane damage (Proske and Morgan, 2001). This may explain the detrimental effects of accentuated eccentric contractions on high speed strength performances detected in the present investigation 48-hour following the exercise session. 


\begin{tabular}{|c|c|c|c|c|c|c|c|c|c|}
\hline \multicolumn{10}{|c|}{ Table 1} \\
\hline \multirow[t]{2}{*}{ Assessment } & \multirow[t]{2}{*}{ Trial } & \multirow[t]{2}{*}{ BL } & \multirow[t]{2}{*}{$15 P$} & \multirow[t]{2}{*}{$24 P$} & \multirow[t]{2}{*}{$48 P$} & \multicolumn{2}{|c|}{ Time Effect } & \multicolumn{2}{|c|}{$\begin{array}{l}\text { Interaction } \\
\text { Effects }\end{array}$} \\
\hline & & & & & & $p$ & $\eta^{2}$ & $p$ & $\eta^{2}$ \\
\hline \multirow{2}{*}{$\begin{array}{l}\text { BTP50 } \\
(\mathrm{W})\end{array}$} & $120 / 80$ & $593.1 \pm 53.2$ & $516.3 \pm 60.4$ & $564.4 \pm 68.4$ & $578.6 \pm 50.1$ & \multirow{2}{*}{0.001} & \multirow{2}{*}{0.657} & \multirow{2}{*}{0.092} & \multirow{2}{*}{0.286} \\
\hline & $80 / 80$ & $598.9 \pm 63.2$ & $562.8 \pm 58.7$ & $586.1 \pm 67.1$ & $591.4 \pm 67.8$ & & & & \\
\hline \multirow{2}{*}{$\begin{array}{c}\text { ISOK25 } \\
(\mathrm{N})\end{array}$} & $120 / 80$ & $1288.7 \pm 151.9$ & $1056.4 \pm 230.3^{* \#}$ & $1167.2 \pm 162.7^{\#}$ & $1252.4 \pm 125.4$ & 0.000 & 0.729 & 0.015 & 0.523 \\
\hline & $80 / 80$ & $1300.5 \pm 135.2$ & $1252.4 \pm 119.6$ & $1237.8 \pm 190.1$ & $1289.7 \pm 148.0$ & \multirow[b]{2}{*}{0.001} & \multirow[b]{2}{*}{0.705} & \multirow[b]{2}{*}{0.025} & \multirow[b]{2}{*}{0.528} \\
\hline \multirow{2}{*}{$\begin{array}{l}\text { ISOK75 } \\
(\mathrm{N})\end{array}$} & $120 / 80$ & $921.2 \pm 156.8$ & $724.9 \pm 119.5^{* \#}$ & $813.2 \pm 104.9^{*}$ & $863.3 \pm 118.6^{\sharp}$ & & & & \\
\hline & $80 / 80$ & $895.7 \pm 157.8$ & $824.0 \pm 107.8^{\#}$ & $838.9 \pm 109.8$ & $851.6 \pm 116.6$ & \multirow{3}{*}{0.001} & \multirow{3}{*}{0.668} & \multirow{3}{*}{0.034} & \multirow{3}{*}{0.346} \\
\hline \multirow{2}{*}{ IBPF (N) } & $120 / 80$ & $2125.1 \pm 223.0$ & $1938.3 \pm 226.2^{* \#}$ & $2075.4 \pm 268.8$ & $2150.6 \pm 224.7$ & & & & \\
\hline & $80 / 80$ & $2166.9 \pm 292.4$ & $2101.7 \pm 273.3$ & $2129.5 \pm 267.5$ & $2149.6 \pm 294.2$ & & & & \\
\hline \multicolumn{10}{|c|}{$\begin{array}{c}\text { BTP50 = bench throw power at } 50 \% \text { of } 1-R M ; I S O K 25=\text { isokinetic peak force at } 25 \\
\mathrm{~cm} / \mathrm{s} ; \mathrm{ISOK} 75=\text { isokinetic peak force at } 75 \mathrm{~cm} / \mathrm{s} ; \mathrm{IBPF}=\text { isometric bench press force; } \\
{ }^{*} \text { indicates a significant }(p<0.01) \text { difference between the two protocols at different } \\
\text { time points (pairwise comparison); indicates a significant }(p \leq 0.05) \text { difference from } \\
\text { BL. All data are reported as mean } \pm S D .\end{array}$} \\
\hline
\end{tabular}
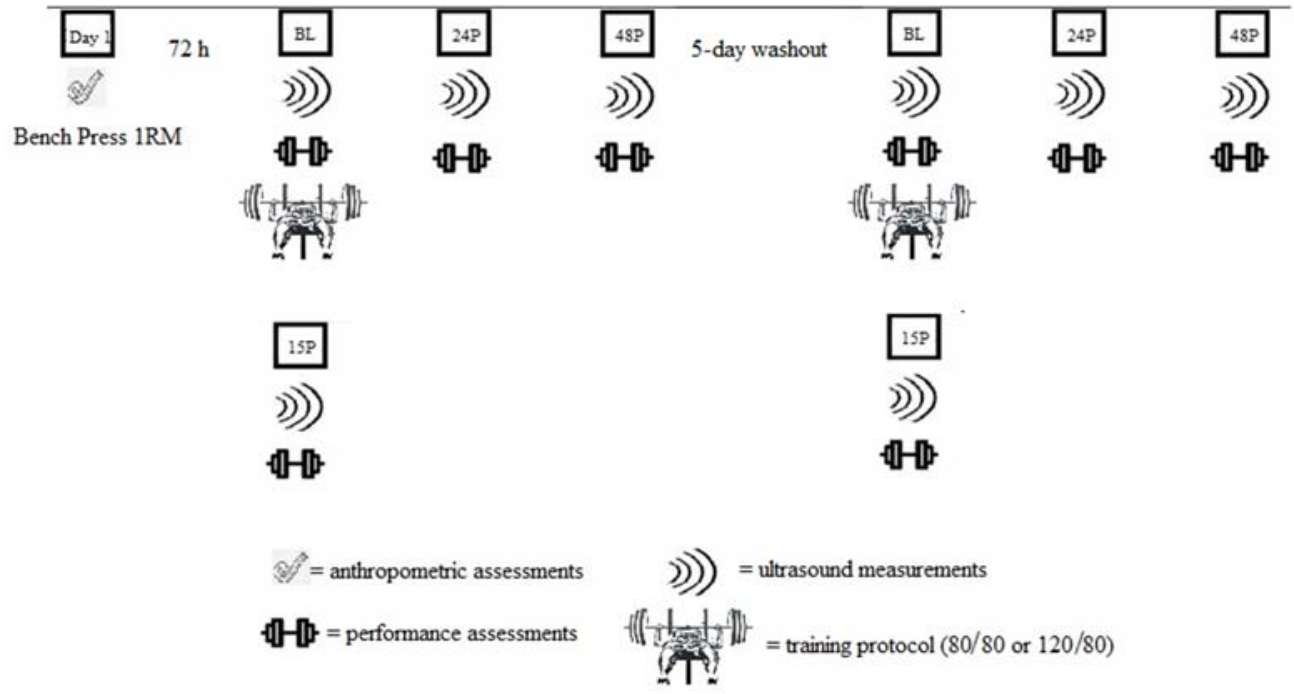

\section{Figure 1}

Experimental protocol of the counterbalanced cross-over research design.

$B L=$ baseline; $15 P=15$ min post; $24 \mathrm{P}=24$ hours post $; 48 \mathrm{P}=48$ hours post. 

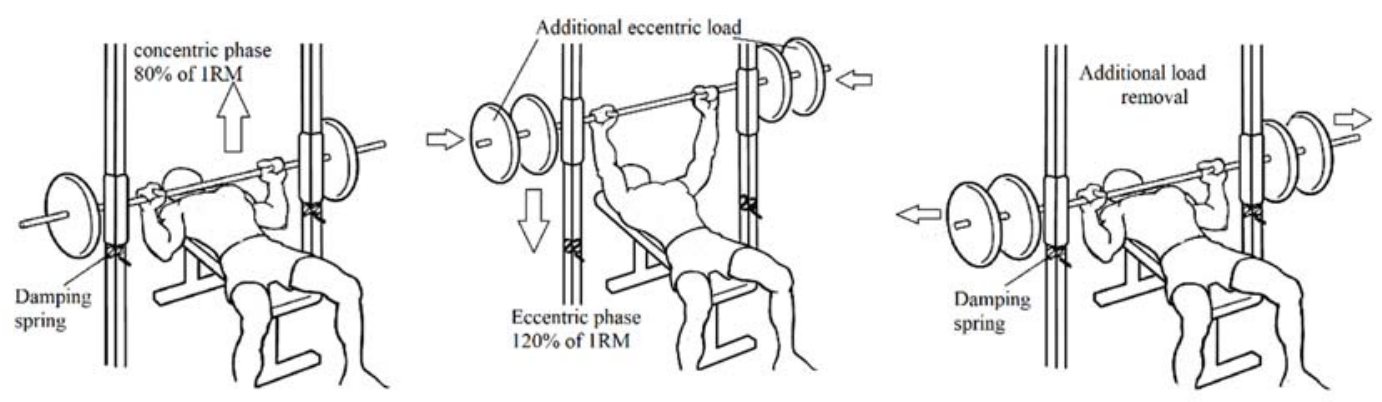

Figure 2

Schematic of the eccentric accentuated protocol (120/80) performed at the Smith machine.

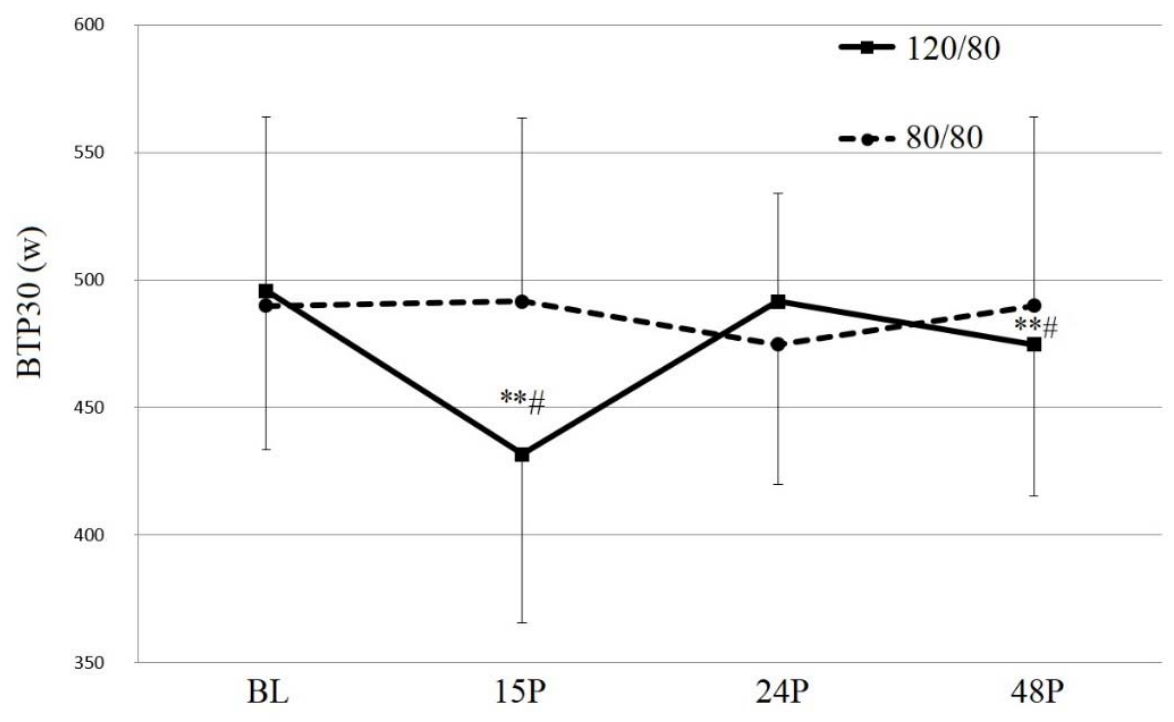

Figure 3

Changes in bench press throw power expressed at 30\% of 1-RM (BTP30) 15-min

(15P), 24-hour (24P) and 48-hour (48P) post-exercise. 120/80 = eccentric

accentuated protocol; $80 / 80=$ regular protocol; ${ }^{*}$ indicates a significant $(p \leq 0.01)$ difference between trials; ${ }^{* *}$ indicates a significant $(p \leq 0.001)$ difference between the two trials; " indicates a significant $(p \leq 0.05)$ difference from BL. All data are reported as mean $\pm \mathrm{SD}$. 


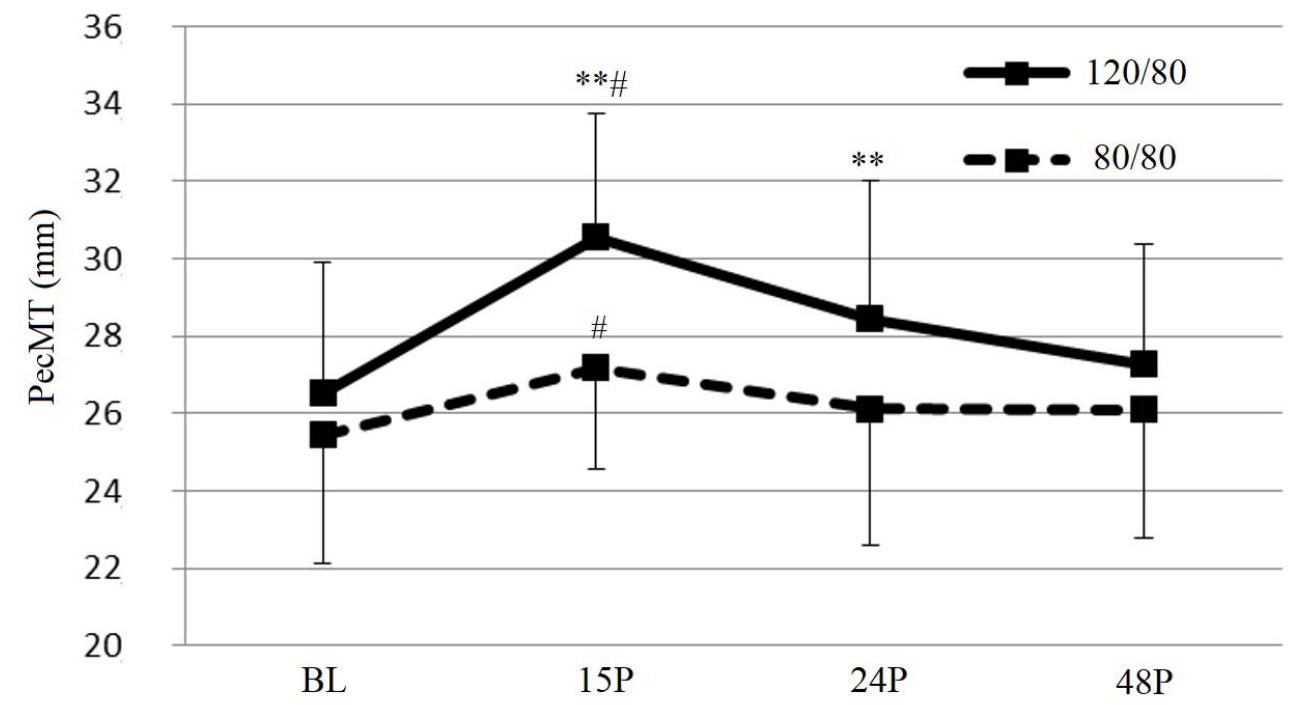

\section{Figure 4}

Changes in PecMT 15-min (15P), 24-hour (24P) and 48-hour (48P) post-exercise.

$120 / 80=$ eccentric accentuated protocol; $80 / 80=$ regular protocol; *indicates a significant $(p \leq 0.01)$ difference between trials; ${ }^{* *}$ indicates a significant $(p \leq 0.001)$ difference between the two trials; $"$ indicates a significant $(p \leq 0.05)$ difference from $B L$. All data are reported as mean $\pm S D$.
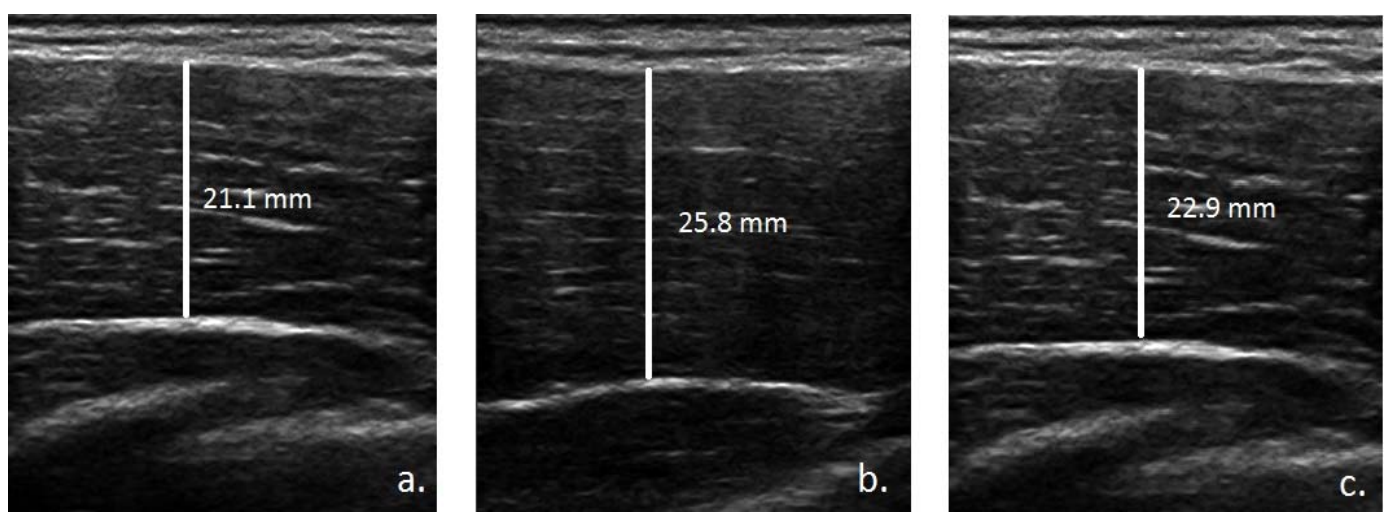

Figure 5

Example ultrasonography images of the pectoralis major (Pec) collected on a participant at BL and $15 P$ following both $120 / 80$ and 80/80 protocols (Figure $5 a, 5 b$ and $5 c$, respectively). 
Significant morphological changes were observed in the pectoral muscle immediately following both $80 / 80$ and $80 / 120$ protocols. However, following the 120/80 exercise protocol the increase in MT was significantly greater $(+15.1 \%)$ than that observed following $80 / 80$ $(+6.9 \%)$. These changes though returned to $\mathrm{BL}$ levels $48 \mathrm{~h}$ following both types of training sessions. Previous investigations have suggested that metabolic stress, local muscle fatigue and reduction in $\mathrm{pH}$, promote muscle post-exercise inflammation and swelling (Dankel et al., 2017; Sjogard et al., 1985). A longer recovery to baseline levels was recently reported following a highvolume resistance exercise session for the lower body (Bartolomei et al., 2017). The relatively quick reduction in swelling of the muscle morphology following both $120 / 120$ and $80 / 80$ training may be related to the lower metabolic stress compared to high-volume exercise protocols (Armstrong et al., 2001). In addition, the experience level of the participants may have provided a degree of protection against eccentric exercise-induced muscle damage from the repeated bout effect (McHugh, 2003). The potential mechanisms involved in the repeated bout effect include changes in motor unit recruitment (Damas et al., 2016), structural remodeling of the extracellular matrix and changes in muscle architecture (Morgan, 1990). A limitation of the present investigation is represented by the possible influence of repeated assessment sessions, including aerobic warm-ups, dynamic and

\section{Acknowledgements}

Funding for this study was obtained from a research grant awarded to ALMA IDEA - University by Bologna.

\section{References}

Abaïdia AE, Delecroix B, Leduc C, Lamblin J, McCall A, Baquet G, Dupont G. Effects of a strength training session after an exercise inducing muscle damage on recovery kinetics. J Strength Cond Res, 2017; 31(1): $115-125$

Abe T, DeHoyos DV, Pollock ML, Garzarella L. Time course for strength and muscle thickness changes following upper and lower body resistance training in men and women. Eur J Appl Physiol, 2000; 81(3): $174-180$

Armstrong RB, Warren GL, Warren, JA. Mechanisms of exercise-induced muscle fibre injury. Sports Med, 1991; 12(3): 184-207

Bartolomei S, Hoffman J, Stout J, Merni F. Effect of lower body resistance training on upper body strength adaptation in trained men. J Strength Cond Res, 2018; 32(1): 13-18

Bartolomei S, Sadres E, Church DD, Arroyo E, Gordon III JA, Varanoske AN, Wang R, Beyer KS, Oliveira LP, Stout JR, Hoffman JR. Comparison of the recovery response from high-intensity and high-volume resistance exercise in trained men. Eur J Appl Physiol, 2017; 117(7): 1287-1298 
Damas F, Nosaka K, Libardi CA, Chen TC, Ugrinowitsch C. Susceptibility to exercise-induced muscle damage: a cluster analysis with a large sample. Int J Sports Med, 2016; 37(8): 633-640

Dankel SJ, Mattocks KT, Jessee MB, Buckner SL, Mouser JG, Loenneke JP. Do metabolites that are produced during resistance exercise enhance muscle hypertrophy?. Eur J Appl Physiol, 2017; 117(11): 2125-2135

Evans EM, Rowe DA, Misic MM, Prior BM, Arngrímsson SA. Skinfold prediction equation for athletes developed using a four-component model. Med Sci Sports Exerc, 2005; 37(11): 2006-2011

Gordon III JA, Hoffman JR, Arroyo E, Varanoske AN, Coker NA, Gepner, Y, Wells AJ, Stout JR, Fukuda DH. Comparisons in the Recovery Response From Resistance Exercise Between Young and Middle-Aged Men. J Strength Cond Res, 2017; 31(12): 3454-3462

Hoffman JR. Physiological Aspects of Sport Training and Performance (Second Edition). Champaign, IL: Human Kinetics, 237-267; 2014

Lieber RL, Shah S, Fridén J. Cytoskeletal disruption after eccentric contraction-induced muscle injury. Clin Ortop Relat Res, 2002; 403: S90-S99

McHugh MP. Recent advances in the understanding of the repeated bout effect: the protective effect against muscle damage from a single bout of eccentric exercise. Scand J Med Sci Sports, 2003; 13(2): 88-97

Morgan DL. New insight into the behavior of muscle during active lengthening. Biopsys J, 1990; 57(2): 209221

Hori N, Newton RU, Nosaka K, Stone MH. Weightlifting exercises enhance athletic performance that requires high-load speed strength. Strength Cond J, 2005; 27(4): 50-55

Proske U, Morgan DL. Muscle damage from eccentric exercise: mechanism, mechanical signs, adaptation and clinical applications. J Physiol Paris, 2001; 537(2): 333-345

Sayers SP, Clarkson PM. Force recovery after eccentric exercise in males and females. Eur J Appl Physiol, 2001; 84(1-2): 122-126

Sakamoto A, Maruyama T, Naito H, Sinclair PJ. Acute effects of high-intensity dumbbell exercise after isokinetic eccentric damage: interaction between altered pain perception and fatigue on static and dynamic muscle performance. J Strength Cond Res, 2010; 24(8): 2042-2049

Schoenfeld B, Grgic J. Eccentric Overload Training: A Viable Strategy to Enhance Muscle Hypertrophy? Strength Cond J, 2017; 40(2): 78-81

Sjogaard G, Adams RP, Saltin B. Water and ion shifts in skeletal muscle in humans with intense dynamic knee extension. Am J Physiol, 1985; 248(2): 190-196

Skurvydas A, Brazaitis M, Kamandulis S. Repeated bout effect is not correlated with intraindividual variability during muscle-damaging exercise. J Strength Cond Res, 2011; 25(4): 1004-1009

Vogt M, Hoppeler HH. Eccentric exercise: mechanisms and effects when used as training regime or training adjunct. J Appl Physiol, 2014; 116(11): 1446-1454

Walker S, Blazevich AJ, Haff GG, Tufano JJ, Newton RU, Häkkinen K. Greater strength gains after training with accentuated eccentric than traditional isoinertial loads in already strength-trained men. Front Physiol, 2016; 7: 149

\section{Corresponding author:}

\section{Sandro Bartolomei}

Department of Biomedical and Neuromotor Sciences

University of Bologna

Bologna 40100 Italy

Tel: 0512094620

E-mail : sandro.bartolomei@unibo.it 